\title{
Higgs masses and couplings in the general 2HDM with unitarity bounds
}

\author{
D. Jurčiukonis* ${ }^{*(1)}$ and L. Lavoura ${ }^{(2)}$ \\ (1) Vilnius University, Institute of Theoretical Physics and Astronomy \\ (2) CFTP, Instituto Superior Técnico, Universidade de Lisboa \\ E-mail: darius.jurciukonisetfai.vu.lt
}

\begin{abstract}
We investigate the general two Higgs doublet model imposing both the unitarity conditions and the bounded-from-below conditions. Both types of conditions restrict the ranges of the parameters of the scalar potential. We study the model in the Higgs basis, i.e. in the basis for the scalar doublets where only one doublet has vacuum expectation value. We use the experimental bounds on the oblique parameter $T$, to produce scalar particles with masses and cubic and quartic couplings of the Higgs in agreement with the phenomenology. The numerical calculations show that the cubic coupling may be up to 1.6 times larger than in the Standard Model, but it may also be zero or even negative. The quartic coupling is always positive and may be up to four times larger than in the Standard Model.
\end{abstract}

The 39th International Conference on High Energy Physics (ICHEP2018)

4-11 July, 2018

Seoul, Korea

\footnotetext{
* Speaker.

${ }^{\dagger}$ D.J. thanks the Lithuanian Academy of Sciences for support through the project DaFi2018.
} 


\section{The model}

The Standard Model (SM) predicts a boson $h_{1}$ which is a scalar and it predicts its cubic and quartic couplings $g_{3}$ and $g_{4}$, which we define through

$$
\mathscr{L}=\cdots-g_{3}\left(h_{1}\right)^{3}-g_{4}\left(h_{1}\right)^{4},
$$

to be $g_{3}^{\mathrm{SM}} \approx 32 \mathrm{GeV}$ and $g_{4}^{\mathrm{SM}} \approx 0.032$, respectively. But Nature could be more complicated than the SM and then $g_{3}$ and $g_{4}$ might have different values. In this paper we analyse the cubic $g_{3}$ and quartic $g_{4}$ couplings of the model with two scalar gauge- $S U(2)$ doublets $\phi_{1}$ and $\phi_{2}$ having the same weak hypercharge. This is usually known as $2 \mathrm{HDM}$. The most general scalar potential is

$$
\begin{aligned}
V= & \mu_{1} \phi_{1}^{\dagger} \phi_{1}+\mu_{2} \phi_{2}^{\dagger} \phi_{2}+\left(\mu_{3} \phi_{1}^{\dagger} \phi_{2}+\text { H.c. }\right) \\
& +\frac{\lambda_{1}}{2}\left(\phi_{1}^{\dagger} \phi_{1}\right)^{2}+\frac{\lambda_{2}}{2}\left(\phi_{2}^{\dagger} \phi_{2}\right)^{2}+\lambda_{3} \phi_{1}^{\dagger} \phi_{1} \phi_{2}^{\dagger} \phi_{2}+\lambda_{4} \phi_{1}^{\dagger} \phi_{2} \phi_{2}^{\dagger} \phi_{1} \\
& +\left[\frac{\lambda_{5}}{2}\left(\phi_{1}^{\dagger} \phi_{2}\right)^{2}+\lambda_{6} \phi_{1}^{\dagger} \phi_{1} \phi_{1}^{\dagger} \phi_{2}+\lambda_{7} \phi_{2}^{\dagger} \phi_{2} \phi_{1}^{\dagger} \phi_{2}+\text { H.c. }\right],
\end{aligned}
$$

where $\mu_{1,2}$ and $\lambda_{1,2,3,4}$ are real.

We use the Higgs basis for the scalar doublets where only $\phi_{1}^{0}$ has VEV

$$
\phi_{1}=\left(\begin{array}{c}
G^{+} \\
v+\left(H+i G^{0}\right) / \sqrt{2}
\end{array}\right), \quad \phi_{2}=\left(\begin{array}{c}
C^{+} \\
\left(\sigma_{1}+i \sigma_{2}\right) / \sqrt{2}
\end{array}\right) .
$$

Here $v$ is the VEV, which is real and positive, and $G^{+}$and $G^{0}$ are (unphysical) Goldstone bosons. In the second equation, $\sigma_{1}$ and $\sigma_{2}$ are real fields and $C^{+}$is the physical charged scalar of the 2HDM.

The unitarity constraints lead to upper bounds on the parameters of the potential. The idea of these constraints is that the scalar-scalar scattering amplitudes at tree-level must respect unitarity. For the 2HDM there are five two-particle scattering channels having different values of the electric charge $Q$ and of the third component of weak isospin $T_{3}$. In order to derive the unitarity conditions one must write the scattering matrices for pairs of one incoming state and one outgoing state with the same $Q$ and $T_{3}$. In order to satisfy those conditions, the eigenvalues of all the scattering matrices should be smaller, in modulus, than $4 \pi$. These conditions were first derived in ref. [1] and the expressions of matrices for our model are presented in ref. [2].

The potential (1.2) must be positive in all the field-space directions for large values of the fields; this means that the scalar potential has to be bounded from below (BFB), which provides stability for the potential. Necessary and sufficient conditions for the scalar potential of the 2HDM to be BFB were derived in ref. [3, 4], which we have implemented in our numerical calculations [2]. Also we apply conditions [5], which guarantee that the vacuum state has a lower value of the potential than all the other possible stability points of the potential.

We emphasize that both the unitarity conditions and the bounded-from-below conditions for the 2HDM are invariant under a change of the basis used for the two doublets. Therefore, one may implement those conditions directly in the Higgs basis.

The mass terms of the scalars are

$$
V=\cdots+\frac{1}{2}\left(\begin{array}{lll}
H & \sigma_{1} & \sigma_{2}
\end{array}\right) M\left(\begin{array}{lll}
H & \sigma_{1} & \sigma_{2}
\end{array}\right)^{T},
$$


with mass matrix

$$
\begin{aligned}
M & =\left(\begin{array}{ccc}
2 \lambda_{1} v^{2} & 2 v^{2} \Re \lambda_{6} & -2 v^{2} \mathfrak{I} \lambda_{6} \\
2 v^{2} \Re \lambda_{6} & M_{C}+\left(\lambda_{4}+\Re \lambda_{5}\right) v^{2} & -v^{2} \mathfrak{I} \lambda_{5} \\
-2 v^{2} \mathfrak{I} \lambda_{6} & -v^{2} \mathfrak{I} \lambda_{5} & M_{C}+\left(\lambda_{4}-\mathfrak{R} \lambda_{5}\right) v^{2}
\end{array}\right) \\
& =R^{T} \times \operatorname{diag}\left(M_{1}, M_{2}, M_{3}\right) \times R,
\end{aligned}
$$

where $R$ is a $3 \times 3$ orthogonal matrix that may be parameterized as

$$
R=\bar{R}_{23}\left(\vartheta_{2}\right) \times \bar{R}_{12}\left(-\vartheta_{1}\right) \times \bar{R}_{23}\left(-\vartheta_{3}\right),
$$

by using rotation matrices $\bar{R}_{12}$ and $\bar{R}_{23}$. The squared mass $M_{1}=(125 \mathrm{GeV})^{2}$ and $M_{2}<M_{3}$. We do not impose any lower limit on $M_{2,3}$; we allow them to be lower than $M_{1}$.

According to equation (1.2) the three-Higgs vertex is given by

$$
\begin{aligned}
g_{3}= & \frac{v}{\sqrt{2}}\left[\lambda_{1} x_{1}^{3}+\left(\lambda_{3}+\lambda_{4}\right) x_{1}\left(1-x_{1}^{2}\right)+x_{1}\left(x_{2}^{2}-x_{3}^{2}\right) \Re \lambda_{5}-2 x_{1} x_{2} x_{3} \mathfrak{I} \lambda_{5}\right. \\
& \left.+3 x_{1}^{2}\left(x_{2} \Re \lambda_{6}-x_{3} \mathfrak{I} \lambda_{6}\right)+\left(1-x_{1}^{2}\right)\left(x_{2} \mathfrak{R} \lambda_{7}-x_{3} \mathfrak{I} \lambda_{7}\right)\right],
\end{aligned}
$$

and the four-Higgs vertex is given by

$$
\begin{aligned}
g_{4}= & \frac{1}{8}\left[\lambda_{1} x_{1}^{4}+\lambda_{2}\left(1-x_{1}^{2}\right)^{2}+2\left(\lambda_{3}+\lambda_{4}\right) x_{1}^{2}\left(1-x_{1}^{2}\right)+2 x_{1}^{2}\left(x_{2}^{2}-x_{3}^{2}\right) \Re \lambda_{5}\right. \\
& \left.-4 x_{1}^{2} x_{2} x_{3} \mathfrak{I} \lambda_{5}+4 x_{1}^{3}\left(x_{2} \Re \lambda_{6}-x_{3} \mathfrak{I} \lambda_{6}\right)+4 x_{1}\left(1-x_{1}^{2}\right)\left(x_{2} \Re \lambda_{7}-x_{3} \mathfrak{I} \lambda_{7}\right)\right],
\end{aligned}
$$

where $x_{k}=R_{1 k}$ for $k=1,2,3$ and $R$ is the matrix in equation (1.6).

\section{Numerical analysis}

The potential (1.2) of the 2HDM, in the Higgs basis, has 11 real parameters: $v, \lambda_{1}, \lambda_{2}, \lambda_{3}, \lambda_{4}$, $\left|\lambda_{5}\right|,\left|\lambda_{6}\right|,\left|\lambda_{7}\right|, \arg \left(\lambda_{6}^{*} \lambda_{7}\right), \arg \left(\lambda_{5}^{*} \lambda_{6} \lambda_{7}\right)$, and $\mu_{2}$. The other three real parameters of the potential depend on these: $\mu_{1}=\lambda_{1} v^{2}$ and $\mu_{3}=\lambda_{6} v^{2}$ ( $\mu_{3}$ is complex, therefore it represents two real parameters). By putting these 11 parameters into the mass matrix (1.5a) we can compute its eigenvalues $M_{2,3}$, diagonalizing matrix $R$, and the Higgs couplings $g_{3}$ and $g_{4}$. A detailed description of this calculation method is presented in ref. [2]. It turns out that this method prefers to produce largish values of the masses and misses lower masses.

In order to have control on the values of the masses, in our numerical work we can use as input different quantities. Besides $v=174 \mathrm{GeV}$ and $M_{1}=(125 \mathrm{GeV})^{2}$, we input $M_{2}, M_{3}, M_{C}, \vartheta_{1}, \vartheta_{2}, \lambda_{2}$, $\lambda_{3}, \Re\left(\lambda_{6} \lambda_{7}^{*}\right)$, and $\Re\left(\lambda_{5}^{*} \lambda_{6} \lambda_{7}\right)$. The angle $\vartheta_{1}$ is in either the first quadrant or the fourth quadrant, with a requirement that $c_{1} \equiv \cos \vartheta_{1}>0.9$ so that the $h_{1} W^{+} W^{-}$coupling is within $10 \%$ of its SM value. The angle $\vartheta_{2}$ is in the first quadrant, corresponding to a choice of the signs of the fields of the new scalars $h_{2}$ and $h_{3}$.

We compute the oblique parameter $T[2,6]$ and check that it is in its experimentally allowed domain [7] $-0.04<T<0.20$. From eq. (1.5) we then compute parameters $\lambda_{1}, \lambda_{4}, \lambda_{5}, \lambda_{6}$, and $\lambda_{7}$. Now we have all the parameters of the model and we require them to satisfy both the unitarity conditions and the BFB conditions. Numerically diagonalizing the mass matrix (1.5a), we find the 


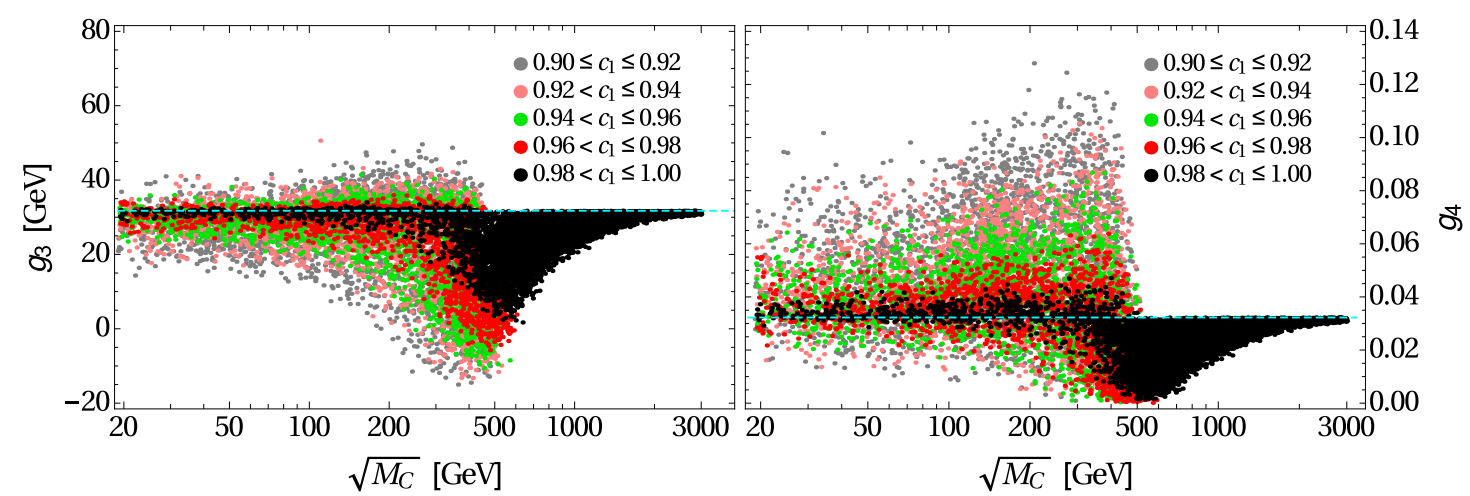

Figure 1: The three-Higgs coupling $g_{3}$ (left panel) and of the four-Higgs coupling $g_{4}$ (right panel)versus $\sqrt{M_{C}}$ in the $2 \mathrm{HDM}$ for various values of $c_{1}$. The dashed lines mark the values of the couplings in the SM.

diagonalization matrix $R$ and we choose the overall sign of $R$ such that $R_{11} \equiv c_{1}>0$. Having matrix elements $R_{11}, R_{12}$, and $R_{13}$ we compute the cubic (1.7) and quartic (1.8) Higgs couplings.

In our numerical work we have found that for $c_{1} \lesssim 0.99$, the masses of the new scalar particles $\left(\sqrt{M_{C}}, \sqrt{M_{2}}\right.$, and $\left.\sqrt{M_{3}}\right)$ of the $2 \mathrm{HDM}$ can be no larger than $\sim 700 \mathrm{GeV}$. For $c_{1} \lesssim 0.95$, they can be no larger than $\sim 550 \mathrm{GeV}$. When $c_{1}$ becomes close to 1 , the masses of all three new scalars may grow strongly to the order of $\mathrm{TeV}$ and in that case they become almost identical.

In figure 1 we have plotted the three- and four-Higgs couplings $g_{3}$ and $g_{4}$ against the mass of the charged scalar $\sqrt{M_{C}}$. One sees that $g_{3}$ may be up to 1.6 times larger than in the SM, but it may also be zero or even negative. On the other hand, $g_{4}$ is always positive because of the boundedness from below of the potential and may be up to four times larger than in the SM. For the masses of the new scalars up to $125 \mathrm{GeV}, g_{3}$ has values in the range $0.3 \lesssim g_{3} / g_{3}^{\mathrm{SM}} \lesssim 1.6$ while $g_{4}$ has values in the range $0 \lesssim g_{4} / g_{4}^{\mathrm{SM}} \lesssim 3$. For the masses up to $500 \mathrm{GeV}$ the couplings reach their maximal values but when the masses of the new scalars grow beyond $1 \mathrm{TeV}$ the couplings $g_{3}$ and $g_{4}$ approach their SM values.

For a more detailed description of this model and for a comparison of the couplings $g_{3}$ and $g_{4}$ in the 2HDM with couplings calculated in other extensions of the SM (Standart Model with the addition of one real singlet, SM with two real singlets, and 2HDM with the addition of one real singlet), we suggest a look at ref. [2].

\section{References}

[1] I. F. Ginzburg and I. P. Ivanov, Phys. Rev. D 72 (2005) 115010 [hep-ph/ 0508020 ].

[2] D. Jurčiukonis and L. Lavoura, arXiv: 1807.04244.

[3] M. Maniatis, A. von Manteuffel, O. Nachtmann, and F. Nagel, Eur. Phys. J. C 48 (2006) 805 [hep-ph/0605184].

[4] I. P. Ivanov and J. P. Silva, Phys. Rev. D 92 (2015) 055017 [arXiv:1507.05100 [hep-ph] ].

[5] Xun-Jie Xu, Phys. Rev. D 95 (2017) 115019 [arXiv: 1705.08965 [hep-ph] ].

[6] W. Grimus, L. Lavoura, O. M. Ogreid, and P. Osland, J. Phys. G 35 (2008) 075001 [arXiv:0711.4022 [hep-ph]].

[7] C. Patrignani et al. [Particle Data Group], Chin. Phys. C 40 (2016) 100001. 